\section{RasGrf1 and Aging}

Kelvin J. A. Davies ${ }^{1,2}$ and Henry Jay Forman ${ }^{1,3}$

Comment on: Borras C et al. Aging. 2011; 3:262-276.

This important article by Borrás et al. demonstrates several interesting and surprising findings [1]. The most intriguing finding was that the complete elimination of normal RasGrf1 increased both average and maximal longevity independent of a role in cancer. We found this to be surprising because RasGrf1, a $\mathrm{Ca}^{2+} /$ calmodulindependent Ras-guanine-nucleotide-releasing factor, would be expected to have a role in normal physiology [2].

The effect of RasGrf1 deletion on aging, which is also accompanied by lower frailty and retention of motor control, appears to be mediated by greater protection against oxidative damage as observed by lower brain lipid peroxidation, liver protein oxidation and maintenance of the brain and liver glutathione redox potential. We must note that the use of malondialdehyde levels as a measure of overall lipid peroxidation in whole tissues is rather suspect and subject to numerous artifacts; nevertheless, the approximate $25 \%$ lower MDA levels in RasGrf ${ }^{/-}$mice (in comparison with wild-type mice) were significant. RasGrf

- mice also exhibited lower levels of oxidized proteins (carbonyl assay) than did wild-type animals which might be due either to lower rates of production, or higher degradation rates of oxidized proteins, or both. It should be noted that old wild-type animals also exhibited lower levels ( $\approx 20 \%$ ) of oxidized liver proteins than seen in wildtype mice. This is surprising since levels of oxidized (typically aggregated and cross-linked) proteins usually increase with age, as Proteasome [3-5] and Lon protease $[6,7]$ activities decline. Indeed, the authors themselves note this expectation in the Results section. Nevertheless, it is impressive that the old RasGrf1 deletion mutants exhibited almost 30\% lower levels of oxidized liver proteins than did the young wild-type mice.

RASGRF1 is expressed in pancreatic $\beta$-cells where it regulates $\beta$-cell mass. So the effects on glucose metabolism is unsurprising. RasGrf1 is also expressed in the hippocampus and hypothalamus is involved in learning and memory. So, one wonders how a human without RasGrf1 would be able to do those functions and whether living longer and stronger might be accompanied by not remembering why one cared.

Nonetheless, the effect on longevity is remarkable and delving deeper in to how the absence of RasGrf1 produces this dramatic effect is worthwhile. For example, it would be interesting to determine whether the ability to increase glutathione in response to stress, which declines in aging, is also maintained in the RasGrf1 knockout mice. Certainly, it would be worth testing the effect of RasGrf1 deletion on Proteasome and Lon levels and stressinducibility.

Another interesting point raised by the authors is that RasGrf1, which is an exclusively paternal allele imprinted gene, suggests that only the male parent determines the effect of this gene's expression on longevity. In several ways, the Ras $G r f 1^{-/-}$mice metabolically resemble mice fed a calorie-restricted diet. One exciting outcome of these studies is that RasGrf1 may be a potential target for design of agents that prolong lifespan and healthspan without the obvious difficulty of restricting caloric intake.

${ }^{1}$ Ethel Percy Andrus Gerontology Center of the Davis School of Gerontology; and ${ }^{2}$ Division of Molecular \& Computational Biology, Department of Biological Sciences of the Dornsife College of Letters, Arts \& Sciences, the University of Southern California, Los Angeles, CA 90089, USA

${ }^{3}$ University of California at Merced, Merced, CA 95343, USA Received: 05/23/11; Published: 05/26/11

1. Borras C et al. Aging. 2011; 3:262-76.

2. Krapivinsky G et al. Neuron. 2003; 40:775-84.

3. Fucci L et al. Proc Natl Acad Sci U S A 1983; 80: 1521-1525.

4. Sitte $\mathrm{N}$ et al. FASEB J. 2000; 14: 1490-1498.

5. Friguet B et al. Ann N Y Acad Sci 2000; 908; 143-154.

6. Bota DA and Davies. Nat Cell Biol. 2002; 4:674-680.

7. Bota DA et al. FEBS Lett. 2002; 532:103-106. 